\title{
Diagrammatic Language for Artificial Intelligence: Representation of Things that Flow
}

\author{
Sabah Al-Fedaghi \\ Computer Engineering Department \\ Kuwait University \\ Kuwait
}

\begin{abstract}
This paper utilizes a diagrammatic language for expressing certain philosophical notions, such as possible worlds, beliefs, and propositions. The focus is on a diagrammatic representation that depicts "things" to show how their various important properties and relations can be explicated in terms of diagrams. The paper does not add a new contribution to philosophy (what is said in it); rather, it contributes a representation tool for philosophy. Akin to specifications in software engineering, the proposed is to provide a complementary technique for expressing different aspects of the involved philosophical concepts that are typically presented in the form of textual explanations. The resultant diagrams seem to be a viable tool that can be utilized in teaching, in communication, and to facilitate an understanding of philosophical problems.
\end{abstract}

Keywords-diagrammatic representation; philosophical diagrammatic language; possible worlds

\section{INTRODUCTION}

It has been a major objective of many philosophers and scientists to achieve a representation without ambiguity through building universal languages. One attempt in this direction is to represent things with symbols instead of words, with the addition of some grammatical rules; this was thought to be sufficient for expressing our conceptions [1]. A representation in this sense is a sign about things. A diagram of a man is a representation because it is about the man. In psychology, a representation is described as having a referent that can be represented in different ways (content and media) [2] or similar terms. Some versions of the so-called representational theory of mind (not of direct concern in this paper) explain the mind in terms of representations [3]. Representation can also be viewed as a relationship between that which represents (sign) and that which is represented [4].

In this paper, the term representation refers to “appearances” (public representation [4]), such as expressions in natural language, algebraic formulae, graphs, or geometric figures [5]. The focus is on a diagrammatic representation that depicts things (will be defined later) that "may exist in a possible or fictional world" [6]. In resemblance to Von Eckard's definition of mental representation, the representation of concern, here, includes such things as "concrete objects, sets, properties, events, and states of affairs in this world, in possible worlds, and in fictional worlds as well as abstract objects such as universals and numbers; that can represent both an object (in and of itself) and an aspect of that object (or both extension and intension); and that can represent both correctly and incorrectly" [7]. Such a representation is utilized "to characterize the kind of phenomena that occur in any knowledge process or that constitute it" [8]. This type of representation is "the means through which a person can externalize his/her mental representations in order to make them visible or accessible to others" [5]. It plays "a central role in social exchange, sophisticated communicators such as humans have developed ways to talk and think directly about representational properties such as truth, accuracy, meaning, and entailment” [4]. The resultant description assumes things with representational properties and aims to model them.

The proposed diagrammatic language is applied to the concepts of possible worlds, beliefs, and propositions to demonstrate how various important properties and relations of things can be explicated in terms of diagrams. For example, [9] invoked "the concept of possible worlds in order to give an analysis of what propositions are; to give an explanation as to why they need to be distinguished from the sentences, which may be used to express them; and to provide a method for identifying and referring to particular propositions [things]" [9]. Accordingly, the paper closely follows Bradley and Swartz's [9] discussion of these topics while recasting them in a diagram that reveals additional properties and relations.

The paper does not add a new contribution to philosophy (what is said in it); rather, it contributes to a linguistic varsity of philosophy. It offers a diagrammatic language akin to specifications in software engineering that provides a complementary means of expressing different aspects of the involved philosophical concepts typically presented in the form of verbose explanations. The resultant diagrams can be utilized in teaching, in communication, and to facilitate an understanding of philosophical problems.

[Many students] consider philosophical texts as boring or think they are not clever enough to understand these texts. These views also give an indication why visualization might facilitate the process of understanding: sensual connections may lower the threshold to deal with such texts. [10](italics added)

Recently, many philosophers, psychologists, logicians, mathematicians, and computer scientists have become increasingly aware of the importance of multi-modal reasoning, and much research has been undertaken in the area of non-symbolic, especially diagrammatic, representation systems [11]. 
Visualization techniques used in science and the arts for the advanced analysis of information and theories can and should be similarly used in the humanities. Within the discipline of philosophy, there are both the possibility and the necessity to examine and present ideas using visualization techniques. [12]

The proposed diagrammatic language has been researched and utilized in software engineering and in several other applications [13-18], and for the sake of a self-contained paper, it will be briefly reviewed in the next section. However, the illustrative example of the vision mechanism of humans given here is a new contribution.

\section{FLOWTHING MODEL}

The Flowthing Model (FM) is a representation tool of things that flow, flowthings, which are defined as what can be created, released, transferred, processed, and received in a flow system (see Fig. 1). Hereafter, flowthings may be referred to as things. As will be discussed later, the notion of thing-ness is wider than the notion of physical, mental, and abstract objects. A no-thing is that which is not created, processed, released, transferred, and received, as will be illustrated later.

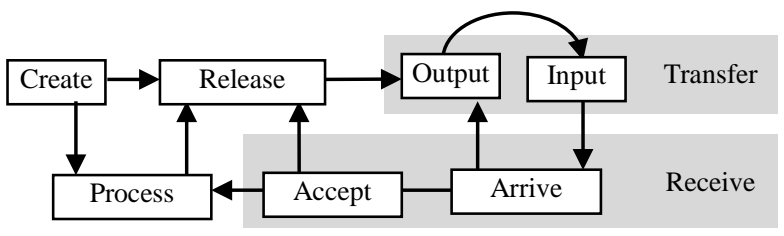

Fig. 1. Flow system

The flow system is an abstract machine used to handle (change though stages) things from their inception or arrival to their de-creation or transmission to the outside. Its stream of flow is a lifeline for things. The stages in Fig. 1 can be described as follows:

Arrive: A thing reaches a new machine.

Accepted: A thing is permitted to enter a machine. If arriving things are always accepted, Arrive and Accept can be combined as a Received stage.

Processed (changed): The thing goes through some kind of transformation that changes it.

Released: A thing is marked as ready to be transferred outside of the machine.

Transferred: The thing is transported somewhere from/to outside of the machine.

Created: A new thing is born or appears in a machine.

FM also uses the notions of spheres and subspheres. These are the "worlds" of things and their flow machines. For example, the actual world is the sphere of all of that is really in it (a human is born, is processed [grows up], and moves from one subsphere to another). A thing is whatever appears in at least one sphere. Note that a sphere can be a thing (e.g., created, processed, ...), e.g., the ship is a sphere of such things as sailors, shipments, supplies, ... machines. Additionally, the ship is a thing that is created (constructed), processed, etc.

When FM represents a portion of reality, spheres, subspheres, flowthings, and triggering are associated with their identifiable counterparts in that portion of reality. Thus, we actually match elements of an FM representation with elements of the target domain. According to Frigg [19]:

A system is a "compact" and unstructured entity and we have to carve it up in order to impose a structure on it. Structures do not really exist until the scientist's mind actually "creates" them or, to put it in a less pretentious way, ascribes them to a system. More specifically, what we have to do is to identify a set of individuals, which can serve as the domain of the structure and then identify a set of relevant relations and operations on this set... Structures are not "ready-made" but result from a way of taking, or demarcating, the system. [Structure] is an imagined physical item, which is equipped with an exactly describable "inner constitution" consisting of a web of properties and their interactions. (Italics added)

The modeler perceived an unstructured reality and processed (carved up) it to trigger the creation of a structured version of reality, such as gazing at a cloud and seeing shapes in it.

Triggering in FM is the activation of a flow, denoted by a dashed arrow. It is a dependency among flows and parts of flows. A flow is said to be triggered if it is activated by another flow (e.g., a flow of electricity triggers a flow of heat) or activated by another point in the flow. Triggering can also be used to initiate events, such as starting up a machine (e.g., remote signal to turn on). Multiple machines captured by FM can interact by triggering events related to other machines in those machines' spheres and stages.

Example: Jin Ma et al. [20] proposed an adapted "Requirement-Function-Behavior-Principle" solution for aiding creative design activities during the conceptual design process. They analyzed the vision mechanism of human to produce summary diagram of functions that is shown in Fig. 2.

[The] human vision system, consists of the basic functions to form vision and the control functions to adjust definition and direction of vision. By processing the visual signal to form instructions to control the incident angle of light and the incident light intensity,... Jin Ma et al. [20]

Such a representation is expressed in a type of transitive verb + noun + complement and input/output flow. A function is discomposed into sub-functions: Adjust the focus, regulate the rotate angle, regulate the light intensity, ...

The purpose of showing Fig. 2 is not to give a fair description of the diagramming method. Rather, the purpose is to display this form of representation that includes heterogeneous shapes. It is difficult to compare the figure with the corresponding FM diagram by listing different shapes and notations, the type of flows and arrows, etc. 


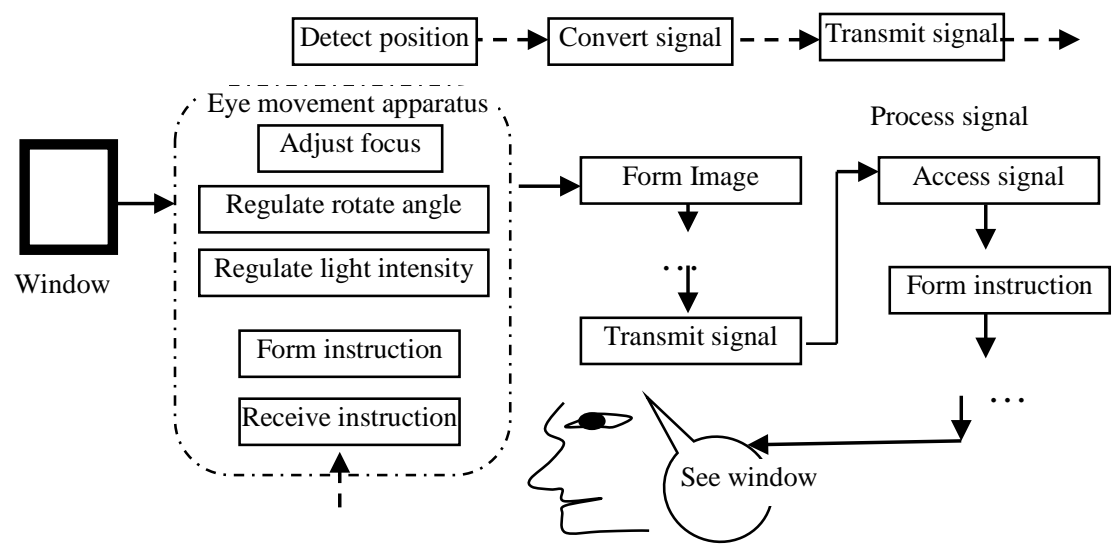

Fig. 2. "Whole flow path of the artificial vision system” (partial figure - re-drawn from Jin Ma et al. [20]

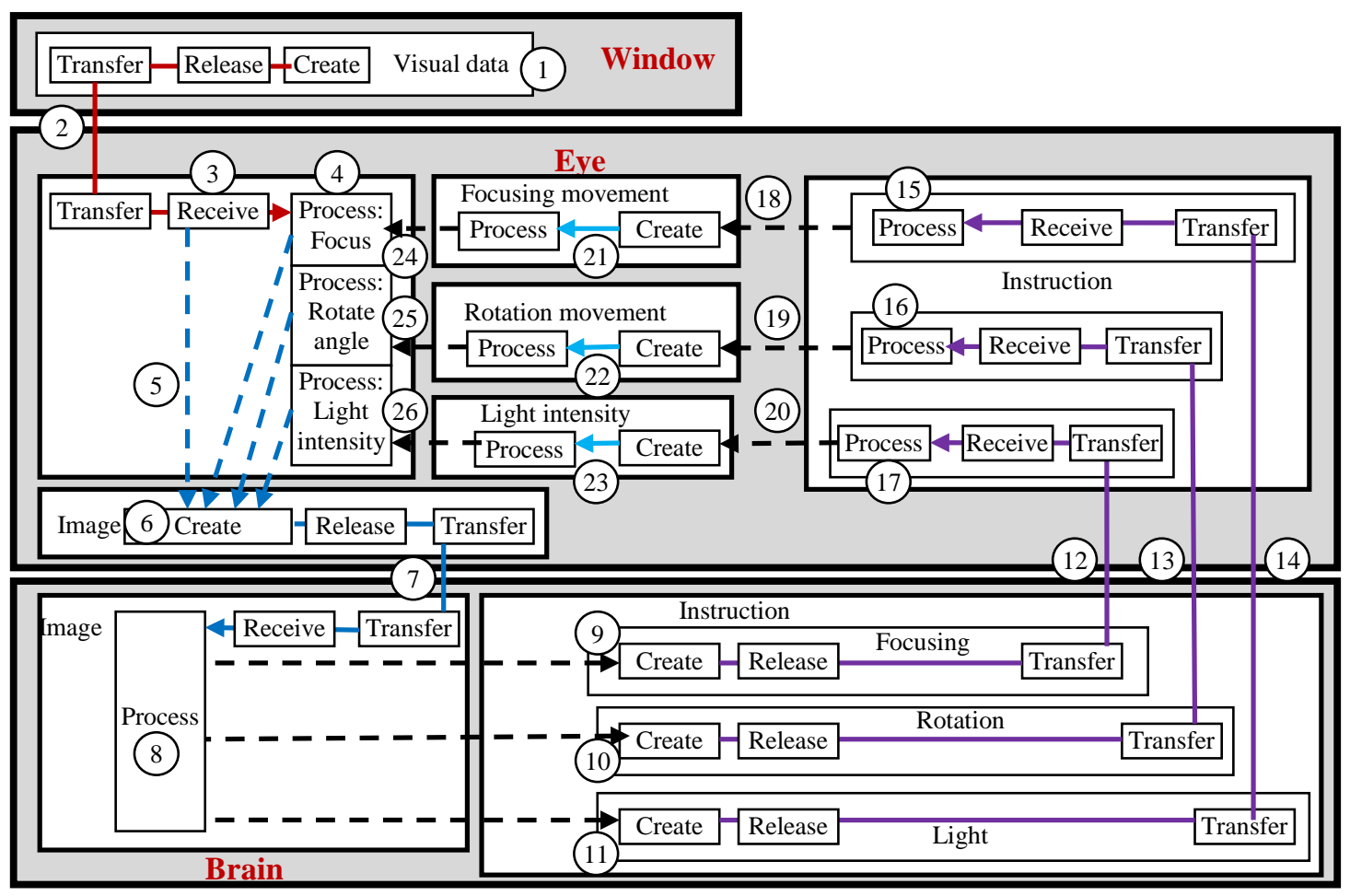

Fig. 3. FM representation of the artificial vision system

A more convenient way is to place the two diagramming representations side by side to contrast their features. Fig. 3 shows the FM diagram that corresponds to a simplified version of the involved system, as we understand it. First, data are generated (circle 1) via the sight of the window, and they flow to the eye (2), where they are received (3) and processed (4). Three types of processed data exist, each placed in a different box to emphasize the differences. According to our understanding, the received raw data trigger (5) the generation (6) of an image that flows to the brain (7), where it is processed (8). The process results in the creation of instructions for:

- Focusing the image (9) and/or

- Rotating the angle of the sight (10) and/or

- Regulating the light's intensity (11)
Then, the instruction(s) flow to the eye (12, 13, and 14), where it (they) is/are processed $(15,16$, and 17$)$ to trigger $(18$, 19 , and 20 ) the creation and processing of the required eye movement (21, 22, and 23). This involves the processing of the incoming data $(24,25$, and 26$)$ to create a refined image (6).

From contrasting Fig. 2 and Fig. 3, such features as the heterogeneity of notions and the uniformity and systematization of each representation appear to be present.

\section{EXPLORING FM REPRESENTATION}

As mentioned previously, this paper focuses on a diagrammatic representation that that can stand for concrete objects in the actual world, in possible worlds, and in fictional worlds, as well as abstract objects. All such things as thoughts, beliefs, desires, perceptions, and imaging are represented as flow things that can be created, processed, received, released, 
and/or transferred. The resultant apparatus also depicts its dynamic trajectory during tits "life" through flow systems. This section explores a variety of these things.

\section{A. Existence}

In Platonic realism, abstract things exist independently of human thinking. The actual world (sphere) is all that really exists: the universe as a whole [9]. An aspect of eexistence in FM can be modelled as a Creation machine in a flow system in a certain sphere.

Consider the status of things such as a gold mountain, which is not real (this example is from Hirst [21] in the context of discussing [22] ideas).

Note that the question is not about the concept or idea of the gold mountain and whether that exists; clearly, it does. But when we say that the gold mountain is 1000 meters tall, we aren't just talking about an idea; it is not the idea that is 1000 meters tall but the alleged thing that the idea is about. [21]

Golden mountain is represented in Fig. 4. There is gold, and there is a mountain. There is also a golden mountain, but it does not exist (has no Create stage) and receives the property (has the property) of receiving (being filled by) gold.

The same representation can be used for the classical example of a round square as shown in Fig. 5. Note that these diagrams are shown to demonstrate that they express the involved notions diagrammatically without any attempt here to discuss the philosophical issues involved with them.

\section{B. Existence, propositions, and sentences}

Propositions are things that are expressible by sentences (e.g., strings of symbols) [9]. Thus, the sentence Fido slept on the mat is a thing that can be created (meaning: there's a sentence), processed (e.g., it is made bold), released, transferred, and received. The referred to (e.g., my dog) Fedo is also a (physical) thing that can be created (e.g., born, appears in the current presentation), processed (e.g., cleaned), .... Additionally, the "meaning" of the sentence is a thing that can be created, processed, .... For example, Fido slept on the mat (the sentence) is created by me, released, transferred, and received by my wife, who processed it to create her understanding of some cause, say, of the dirt on the mat. In this case, the meaning is: At a previous time, my dog, Fido, was received on the mat and then released and transferred from the mat. Accordingly, all of these things - the sentence, (physical) Fido, and meaning-are represented not as mere things but also with their flow among creation in processing, releasing, transferring, and receiving states. These five "flow stoppages in flow (stages)" come inevitably with a thing.

In Fig. 6, the left diagram represents that Fido exists where Fido is represented by the outer box and the stage Create, and that means that There exists Fido is a thing in this presentation. In reality, Fido appears without the tag Create (exists) because the mere appearance of Fido as a physical object, toy, picture, concept, or character in a story implies it exists in that context as a thing. In a representation, this has to be specified explicitly.
In the right diagram of Fig. 6, Fido appears to be a thing that flows through stages to the mat, assuming it was created previously. The point is that a thing always appears in at least one flow stage. The diagrammatic representation refers to the referent and its flow in the given sphere (contexts of flow).

In Fig. 7, a Unicorn does not exist in the actual world (no creation stage), but it exists in Greek methodology, which is in the actual world.

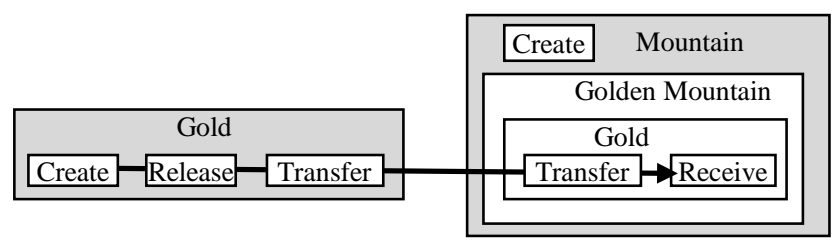

Fig. 4. Golden mountain as a non-existing sphere

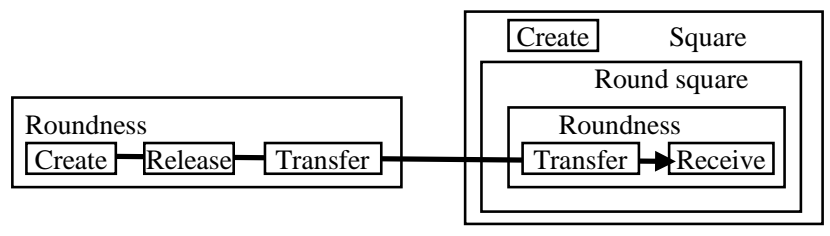

Fig. 5. Round square as a non-existing sphere

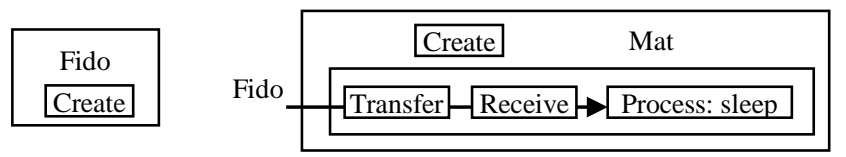

Fig. 6. The thing Fido and possible flows

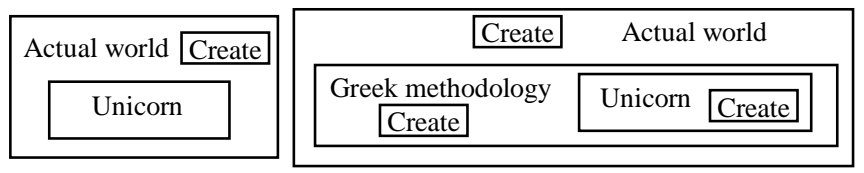

Fig. 7. Non-existence sphere and Unicorn as a thing

The sphere is the background (the frame) where things appear, e.g., painting appears on the surface of a paper and a sound “appears" in air, etc. Accordingly, Fig. 7 (left) says that the Unicorn sphere appears in the sphere of the actual world but it does not exist (no Create stage). That is, it is nothing in the actual world. A non-existent sphere is similar to the outer sphere mentioned previously: It has no stage. When a critic talks about the depth of a painting, he/she is talking about the non-existence sphere. It is the between-the-words sphere, which has no thing. In the right diagram of Fig. 7, the unicorn is a thing created in Greek methodology. Note that the actual world and Greek methodology spheres are things. An existing sphere can be, in its turn, a thing (e.g., created, processed, ...).

\section{Attributes}

In FM, the so-called "attribute" is a thing, e.g., "the Rose is red” is expressed diagrammatically as the sphere Rose, which receives the (flow) Red thing as shown in Fig. 8. The diagram expresses that in some sphere (e.g., actual world - the outer box), there exists (created) a rose that is red. That is, in the sphere under the process of representation, there is the sphere Rose (also a thing since it has the Create stage) that gets the thing's Redness. It is assumed that the outer sphere includes a 
subsphere that creates Red(ness). For simplicity's sake, when appropriate, the outer sphere (box) will not be drawn - Fig. 9 (as we did in the diagrams of the subsection A. Existence). This outer sphere "exists" by representation (it appears in front of our eyes); hence, it is not necessary to insert the Create stage in it. The outer sphere is just a canvas where a painter creates his/her painting, but the canvas is not part of the painter's creation. It is "the spatial aspect ...that provides the raw material for the creation and transformation of diagrams" [23].

Fig. 10 shows a sphere of a red rose and red car. For simplification purposes, the boxes of the subspheres' Red(ness) in Car and Rose (bold circumstance) may be eliminated.

Fig. 11 shows two worlds (drawn as ellipses for illustrative purposes), one with red roses and the other with black roses.

Fig. 12 shows that the black rose is fiction in the actual world (which is, by assumption, the background of the figure).

\section{Beliefs}

According to Bradley and Swartz [9], the term belief is ambiguous in terms of (a) the state, act, or disposition of believing, and (b) that which is believed.

Suppose John Doe believes himself to be ill... In such a case we would be asking about John Doe's state of belief (or, as some would say, his "act of believing"). His belief, in this sense of the word..., is something which may arise at a specific moment of time and persist through time; it may be brought about or caused by some other event or state of affairs, e.g., by his having eaten too much. [9] (italics added)

Fig. 13 shows the FM representation of this state of belief. States of affairs are obtain (ON) or not (OFF) [24]. Thus, a state is either belief or non-belief. Its creation (1), as in the left diagram of Fig. 13, occurs due to illness (2), which is processed (takes its course) and, in turn, is triggered (3) by something. On the other hand, its creation, as in the right diagram, is triggered by something without one's actually being ill. Thus, a state may or may not have a content (illness).

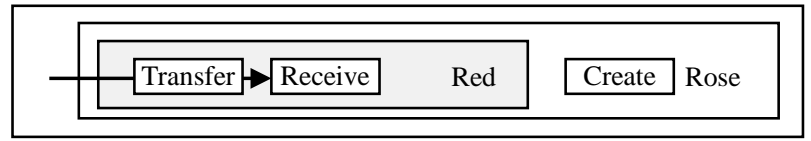

Fig. 8. The sphere Rose receives the Red thing

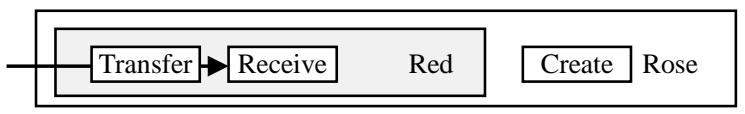

Fig. 9. Simplification of Fig. 8

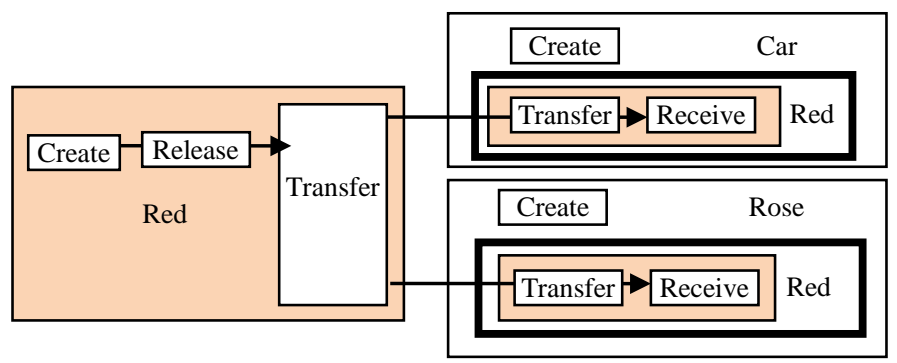

Fig. 10. The spheres of Red Rose and Red Car

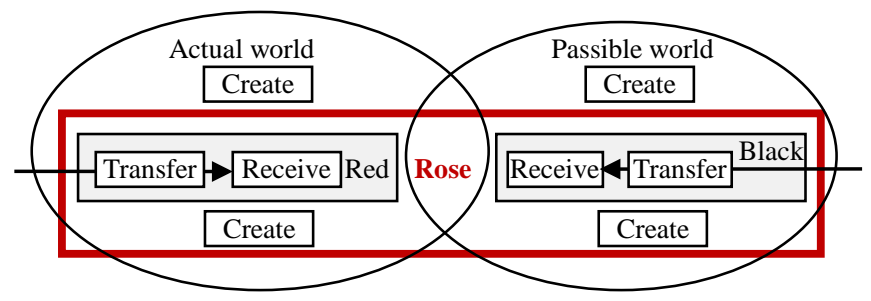

Fig. 11. Two worlds, one with red roses and the other with black roses

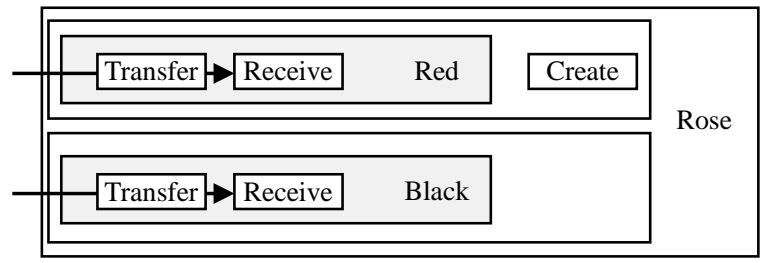

Fig. 12. A black rose is one of things in this world, but it does not exists (no Create stage)
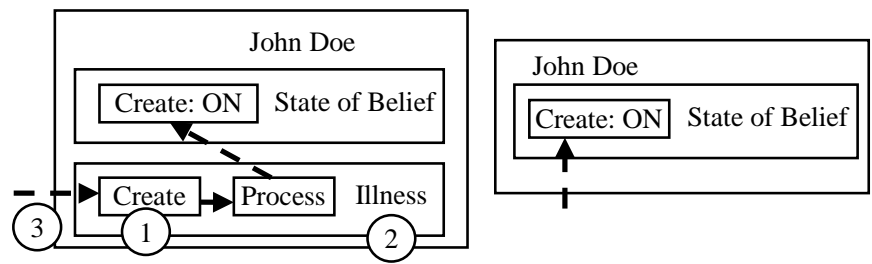

Fig. 13. Two situations that may create State of belief

On the other hand we may distinguish the content of his belief, that which he believes. It is this latter feature which may occur in other persons' beliefs as well. Although no other persons can have John Doe's belief in the sense that their acts of believing cannot be the same act as John Doe's, what they believe, viz., that John Doe is ill, may be shared both by them and John Doe. In this second sense of "belief, the sense in which we talk of what is believed ..., a belief may be shared by many persons. [9] (italics added)

Fig. 14 shows the representation of this case. An actual illness (content) (circle 1) triggers a belief (2) in John Doe. Process (3) means that the illness has taken its course. Thus, the occurrence (creation, existence) of an illness (sooner or later) triggers (4) the generation (5) of an illness sign, which is communicated (flow) to others (6). Processing such a sign (7) triggers (8) the belief in any other person that John Doe is ill.

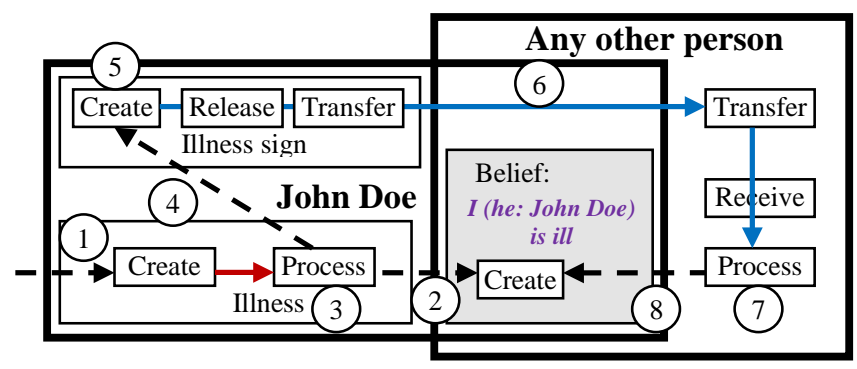

Fig. 14. Sharing belief

The total picture would be further fixed if we introduce truth values as shown in Fig. 15. The belief sphere (circle 1) includes the belief itself (2), its truth value (3), the illness (4), and its truth value (5). Note that the belief (1) is in John Doe, 
and it includes two sub-spheres: itself (2) and its content illness (4). However, the truth value of the belief (3) is not inside John Doe; rather, it exists in abstract. Similarly, the truth value of illness is not in John Doe or his belief but rather in abstract. Let us refer to the truth values by their numbering, 3 and 5 .

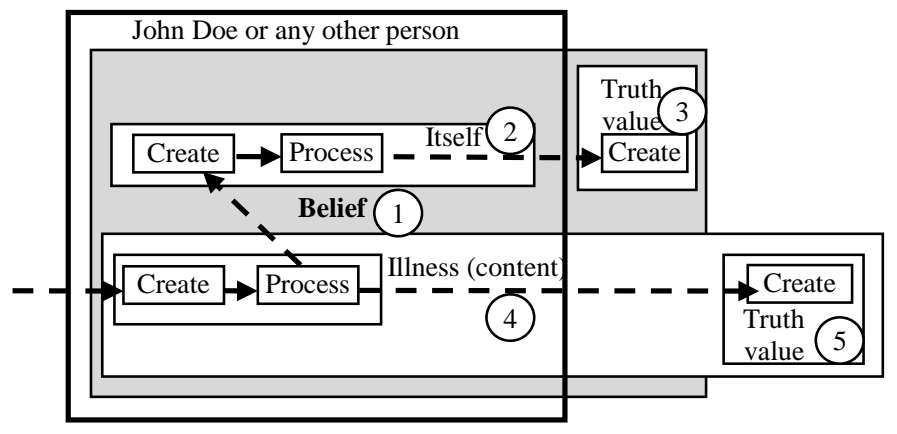

Fig. 15. John Doe, his belief and truth values

\section{CONCLUSION}

This paper has utilized a diagrammatic language for expressing philosophical concepts that potentially can be applied in "diagrammatic thinking and representations" in artificial intelligence. The contribution of this paper is limited to proposing the use of the diagrammatic representation and demonstrating its viability for representing certain philosophical concepts. A great deal of materials has been left out for future work. Nevertheless, the diagrammatic language is worth further discussion and investigation in philosophy that may prove some advantages at least in portraying certain philosophical problems.

\section{REFERENCES}

[1] J. Wilkins, 1614-1672: An Essay Towards a Real Character, and a Philosophical Language. London: Printed for S. Gellibrand and J. Martin, 1668, xx-yy.

[2] Z. D. J. Perner, The Many Problems of Representation. http://www.google.co.th/url?sa $=t \& r c t=j \& q=\& e s r c=s \& s$ ource=web\&cd $=1 \&$ cad $=$ rja\&uact $=8 \& v e d=0$ ahUKEwir58m9xfjMAhWJ PY8KHQo8DW4QFggaMAA\&url=http\%3A\%2F\%2Fwww.lifesci.susse x.ac.uk\%2Fhome\%2FZoltan_Dienes\%2FRepresentation.pdf\&usg=AFQ jCNF4lA8CEuhzFwAPzOwpRvHY15DALA\&bvm=bv.122852650,d.c2 I

[3] K. Sterelny. The Representational Theory of Mind: An Introduction. Location: Blackwell, 1990, xx-yy.

[4] J. G. Greenberg, The Semiotic Spectrum, Ph.D. dissertation, Department of Philosophy, Rutgers University. October 2011

[5] E. F. R. Ledesma, "Representation registers in the solution of calculus problems," Creative Education. Location, vol.2, no.3, pp. 270-275, 2011.

[6] S. Scott, Metarepresentation in Philosophy and Psychology, The $23^{\text {rd }}$ Annual Conference of the Cognitive Science Society, August 2001. http://www.google.co.th/url?sa=t\&rct=j\&q=\&esrc=s\&source=web\&cd= $1 \&$ cad=rja\&uact $=8 \& v e d=0$ ahUKEwjV-

5O3xvjMAhVHRI8KHdh3BpYQFggaMAA\&url=http\%3A\%2F\%2Fcon ferences.inf.ed.ac.uk\%2Fcogsci2001\%2Fpdf-

files\%2F0910.pdf\&usg=AFQjCNE74V6qiTIWbtV12maODY8Wl9dNA
[7] B. Von Eckardt, "Mental representation,” In The MIT Encyclopedia of the Cognitive Sciences, R. A. Wilson and F. C. Keil. Cambridge, Massachusetts: MIT Press, 1999, pp. 527-529.

[8] R. Duval, "A cognitive analysis of problems of comprehension in a learning of mathematics," Educational Studies in Mathematics. Location, vol. 61, no. 1, pp. 103-131, February 2006.

[9] R. Bradley and N. Swartz, Possible Worlds: An Introduction to Logic and Its Philosophy, $4^{\text {th }}$ printing. Location: Hackett Publishing Company, Inc., Year, $\mathrm{xx}-\mathrm{yy}$

[10] V. Reichl and M. M. Keitsch, "Pictorial involvement: Providing access to philosophical texts via visualization through artistic practice," FORMakademisk. Location, vol. 5, no. 2, pp. xx-yy, Year.

[11] Stanford Encyclopedia of Philosophy, Diagrams. Location: Publisher, 2013, xx-yy.

[12] C. F. DiSalvo, "Philosophy and visual representation: Imaging the impossible,” Leonardo Music Journal. Location vol. 32, no. 2, pp. 8386, 1999.

[13] S. Al-Fedaghi, "Diagrammatic representation as a tool in clarifying logical arguments," International Journal of Advanced Research in Artificial Intelligence (IJARAI). Location, vol. 4, no. 10, pp. xx-yy, 2015.

[14] S. Al-Fedaghi, "Computer science approach to information-like artifacts as exemplified by memes," International Journal of Advanced Computer Science and Applications (IJACSA). Location, vol. 6, no. 6, pp. xx-yy, 2015.

[15] S. Al-Fedaghi, "On a flow-based paradigm in modeling and programming," International Journal of Advanced Computer Science and Applications (IJACSA) Thomson-Reuters (ESCI). Location, vol. 6, no. 6, pp. 209-217, 2015.

[16] S. Al-Fedaghi, “Toward flow-based semantics of activities," International Journal of Software Engineering and Its Applications SCOPUS. Location, vol. 7, no. 2, pp. 171-182, 2013.

[17] S. Al-Fedaghi, "Awareness of context and privacy," The American Society for Information Science \& Technology (ASIS\&T) Bulletin SCOPUS. Location, vol. 38, no. 2, pp. xx-yy, 2011.

[18] S. Al-Fedaghi, Schematizing Proofs Based on Flow of Truth Values in Logic, IEEE International Conference on Systems, Man, and Cybernetics (IEEE SMC 2013), October 13-16, Manchester, UK.

[19] R. Frigg, "Models and Representation: Why Structures Are Not Enough," Measurement in Physics and Economics Project Discusion Paper Series, DP MEAS 25/02, London School of Economics, 2002.

[20] J. Ma, J. Hu, K. Zheng, and Y. Peng, "Knowledge-based functional conceptual design: Model, representation, and implementation," Concurrent Engineering: Research and Applications. Location, vol. 21, no. 2, pp. 103-120, 2013.

[21] G. Hirst, "Existence assumptions in knowledge representation," Artificial Intelligence. Location, vol. 49, pp. 199-242, 1991.

[22] A. Meinong and O. Gegenstandstheorie, in A. Meinong, Ed., Untersuchungen zur Gegenstandstheorie und Psychologie (Barth, Leipzig, 1904); reprinted in A. Meinong, Gesamtausgabe, vol. II (R. Hailer and R. Kindinger, Eds.) (Akademische Druck und Verlaganstalt, Graz, 1969-1978), pp. 481-535; reprinted as "The theory of objects" (I. Levi, D. B. Terrell, and R. M. Chisholm, translators), in R. M. Chisholm, Ed., Realism and the Background of Phenomenology (Glencoe, IL: Free Press, 1960, pp. 76-117).

[23] K. Fathulla, "Understanding diagrams: A pointer to the development of diagramming software," Visible Language. Location, vol. 42, no. 3, pp. $\mathrm{xx}-\mathrm{yy}, 2008$.

[24] Stanford Encyclopedia of Philosophy, States of Affairs. Location: Publisher, 2012, http://plato.stanford.edu/entries/states-of-affairs/ 